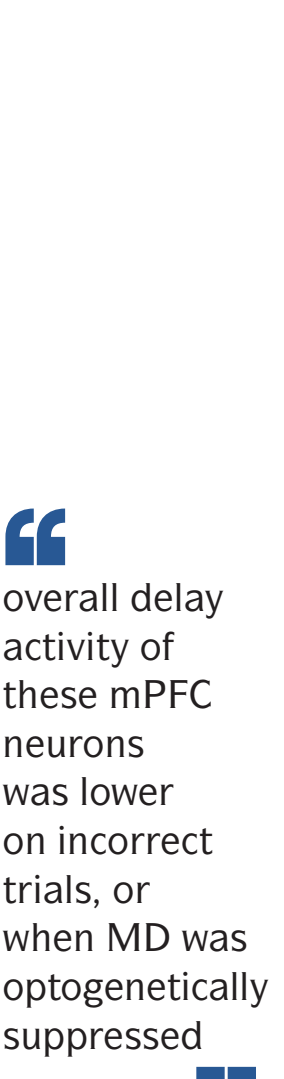

כ5

WORKING MEMORY

\title{
Persistence is key
}

Persistent cortical activity during the delay between different phases of a behavioural task - known as 'delay activity' - maintains taskbased information in the absence of sensory input; however, its circuit basis is unclear. Three new studies now implicate thalamocortical connections in the maintenance of delay activity in mice.

In the first study, Bolkan et al. trained mice in a task in which they explored one arm of a T-maze and then, after a 60-second delay, were rewarded for going into the opposite arm. Optogenetic inhibition of projections from the mediodorsal thalamus (MD) to the medial prefrontal cortex (mPFC) during the delay phase impaired performance, as did inhibition of $\mathrm{mPFC}$ projections to the MD during the choice phase. These results suggest that the MD supports the maintenance of information in the MPFC during the delay, whereas output from the $\mathrm{mPFC}$ to the MD during the choice phase guides action.

Further analysis revealed that some mPFC neurons showed transient increases in activity during the delay periods. The timing of these activity bursts in different neurons was distributed to cover ('tile') the entire delay period. Interestingly, the overall delay activity of these MPFC neurons was lower on incorrect trials, or when the MD was optogenetically suppressed, implying that the MD is crucial for sustaining cortical delay activity. Consistent with this finding, enhancing MD excitability during the delay improved task performance.

In the task used by Schmitt et al., a high-pass or low-pass filtered white noise signalled a 'rule' about which of two stimuli presented simultaneously after a delay the mice had to attend in order to obtain a reward. Some putative pyramidal neurons in the PFC showed transient, rule-selective responses that, as in Bolkan et al.'s study, tiled across the delay period.

Schmitt et al. found that optogenetic inhibition of the MD during the delay suppressed responses in the PFC and impaired task performance, suggesting that the MD enables rule maintenance in the PFC. The authors hypothesized that the MD may act by increasing functional connectivity among PFC pyramidal neuron networks. Indeed, upregulating MD excitability increased the functional connectivity of putative PFC pyramidal neurons without changing their overall spiking rate, augmented PFC rule-specific representations and improved task performance. By contrast, driving spikes in the PFC diminished rule specificity and performance. Importantly, MD neurons were rule non-selective but were sensitive to task context, suggesting that their enhancement of functional PFC connections may be task specific.

In the third study, by Guo et al., mice explored an object using their whiskers and, after a brief delay, reported its location by licking to the left or right. During the delay period, neurons in the anterior lateral motor cortex (ALM) showed 'preparatory' activity - a form of delay activity that represents future actions. Moreover, neurons in the thalamic nuclei that innervate the $\operatorname{ALM}\left(\right.$ thal $_{\mathrm{ALM}}$ ) also showed selective preparatory activity, and unilateral optogenetic inhibition of the ALM or thal $\mathrm{ALM}_{\mathrm{AL}}$ during the delay impaired task performance when the animal had to lick the contralateral side.
Photostimulation of GABAergic cells in the thal $\mathrm{ALM}_{\mathrm{A}}$ strongly reduced both local activity and excitatory ALM activity. By contrast, weak optogenetic inhibition of the thal $\mathrm{ALM}_{\mathrm{AL}}$ caused a decrease in selectivity in the ALM neurons, with only a small change in overall activity level. This suggests that the thal ${ }_{\mathrm{ALM}}$ does not provide a uniform input to all ALM neurons but may contribute to their selectivity in preparatory activity.

Together, these studies demonstrate a key role for the thalamus in generating persistent delay activity that represents rules, locations or motor actions in different tasks. This supports the idea that the thalamus does not simply 'relay' information to the cortex but directly influences cognition.

Natasha Bray

ORIGINAL ARTICLES Bolkan, S. S. et al. Thalamic projections sustain prefrontal activity during working memory maintenance. Nat. Neurosci. http://dx.doi.org/10.1038/nn.4568 (2017)| Schmitt, L. I. et al. Thalamic amplification of cortical connectivity sustains attentional control. Nature 545, 219-233 (2017) |Guo, Z. V. et al. Maintenance of persistent activity in a frontal thalamocortical loop. Nature 545, 181-186 (2017) 